\title{
AUA1: New immunocytochemical marker for detecting epithelial cells in body cavity fluids
}

\author{
G Kocjan, E Sweeney, KD Miller, L Bobrow
}

\begin{abstract}
Further to detailed genetic and biochemical characterisation of AUA1 as a surface glycoprotein present on epithelial cells, the antibody against AUA1 was used as an immunocytochemical marker of epithelial cells in body cavity fluids in an attempt to improve the diagnosis made on routine staining. AUA1 was initially tested in 144 morphologically clear cut effusions. It was positive in 46 of $52(88 \%)$ carcinomas and negative in 82 of $84(98 \%)$ benign effusions, including technically inadequate or poorly cellular preparations. There were no false positive results. AUA1 was subsequently used more selectively-that is, in 42 of 175 (24\%) of morphologically difficult fluids. AUA1 provided essential diagnostic information in 15 of $42(36 \%)$ and confirmed diagnosis in 17 of $42(40 \%)$, thus enabling accurate diagnosis in a further 32 of $42(76 \%)$ of the difficult cases. The total diagnostic accuracy was therefore 94.3\%.
\end{abstract}

AUA1 is a reliable immunocytochemical marker for detecting epithelial cells in body fluids. Its use improves diagnostic accuracy of morphological assessment in difficult cases.

AUAl is a human cell surface antigen defined by the monoclonal antibody AUA1. It was raised conventionally by Arklie (1981) by immunising $\mathrm{BALB} / \mathrm{c}$ mice with the colonic adenocarcinoma cell line LoVo. The genetic locus for this antigen has been assigned to chromosome 2. The product of the gene MIC 18 is a single 35 kilodalton protein expressed by a limited set of normal epithelial cells and a wide variety of epithelial tumours. ${ }^{1}$ Further to the successful application of this antibody as an immunohistochemical marker to histological sections of various epithelia, ${ }^{12}$ we were interested to establish whether AUA1 could be used as an aid in the detection of epithelial cells in body cavity fluids. Particularly encouraging was the fact that, unlike the antibodies to low molecular weight keratins, such as CAM 5.2, AUA1 does not stain normal mesothelium.

\section{Methods}

Initially we used AUAl (Unipath Ltd) as an immunocytochemical marker in addition to routine staining of 144 morphologically diag- nosed effusions (80 pleural and 64 peritoneal). Slides for immunocytochemistry were kept unfixed (air dried), stored at $-20^{\circ} \mathrm{C}$ and were stained according to the alkaline phosphataseantialkaline phosphatase (APAAP) method modified for cytological preparations. More recently, we have used AUA1 more selectively-in 42 of $175(24 \%)$ of fluids where morphological diagnosis was difficult and was confirmed by either poor clinical outcome or by tissue biopsy.

\section{Results}

Comparison of the morphological assessment with AUA1 staining in 144 of the morphologically clear cut cases showed that AUA1 was positive in 46 of $52(88 \%)$ of the malignant effusions and negative in 82 of $84(98 \%)$ of benign effusions, including those which were technically inadequate or poorly cellular. The two positively stained benign effusions

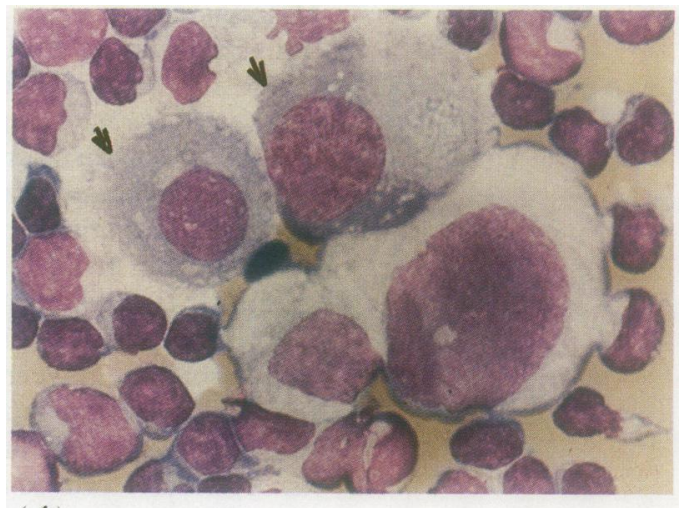

(A)

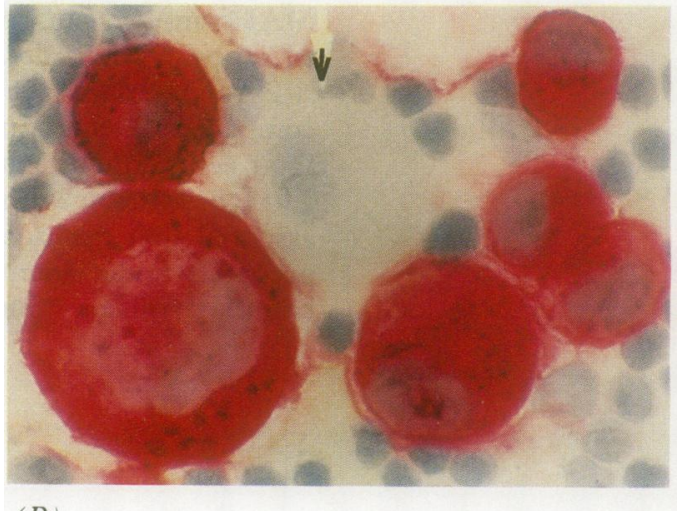

(B)

Pleural effusion containing cells from a metastatic breast carcinoma. (A) Malignant cells; mesothelial cells are indicated with arrows (May-Grünwald-Giemsa, oil immersion ); (B) $A U A 1$ positive staining with $A P A A P$ highlights the malignant cells. Mesothelial cell is negative (arrow) (oil immersion). 
contained cells from the fallopian tube and ovarian adenofibroma, respectively.

In the subsequent series of 42 diagnostically difficult cases (benign and malignant) AUA1 provided essential diagnostic informationthat is, that which could not have been reached by morphology alone-in 15 of 42 $(36 \%)$, and confirmed morphological diagnosis in 17 of $42(40 \%)$ cases, thus enabling accurate assessment in a further 32 of 42 $(76 \%)$ difficult cases, bringing the total diagnostic accuracy to $94 \cdot 3 \%$. Malignant effusions included two mesotheliomas, an osteosarcoma, and a makignant schwannoma, none of which stained with AUA1. Morphological appearances in all difficult cases were reviewed and compared with immunocytochemistry.

\section{Discussion}

Conventional cytological diagnosis of malignant cells in effusions has its limitations, ranging from $15 \%-27 \%$ false negative reports. ${ }^{34}$ Various techniques, including conventional histochemistry, morphometry, electron microscopy and immunocytochemistry, are all used in an attempt to discriminate between benign and malignant cells and also between mesothelioma and peripheral pulmonary adenocarcinoma. ${ }^{5}$ Wick et al found that, although specific, periodic acid Schiff-diastase stained only $63 \%$ of the peripheral adenocarcinomas while hyaluronidase sensitive colloidal iron positivity, although restricted to mesotheliomas, stained only $41 \%$ of cases. The most commonly used immunocytochemical markers in effusions are cytokeratins, epithelial membrane antigen (EMA), carcinoembryonic antigen (CEA), and oncofetal protein $B 72 \cdot 3$. Cytokeratins and EMA stain both mesothelium and epithelium with varying intensity. CEA shows high specificity but variable sensitivity, ranging from $68 \%-96 \%$ for malignant effusions. ${ }^{56}$ B72.3 positivity for malignant epithelial cells ranges between $84 \%{ }^{5}$ and $90 \%{ }^{7}$ Ber EP4 has been successfully characterised on histological material and, like AUA1, seems to be highly specific for epithelial cells although, in our preliminary experience, less sensitive.

In the light of our own experience and that of the others, ${ }^{8}$ we have recently stopped using a panel of antibodies on our effusions and are using only AUA1 instead.

Caution should be exercised in the interpretation of positive AUAl staining of benign epithelial cells in peritoneal washings and effusions. These can originate from epithelia of the fallopian tube or from foci of pelvic endometriosis. ' We have found positively stained epithelial cells originating from ovarian adenofibroma. Particularly useful is the absence of staining in malignant mesothelioma. Although there are only two cases in this series, we are encouraged by the experience described with histological material from this department. ${ }^{10}$

In conclusion, AUAl is the most sensitive of the currently available immunocytochemical markers for detection of epithelial cells in body fluids, and for the distinction between benign and malignant mesothelial cells. Combined with morphological assessment it can improve diagnostic accuracy.

1 Spurr NK, Durbin H, Sheer D, Parkar M, Bobrow L, Bodmer WF. Characterisation and chromosomal assignment of a human cell surface antigen defined by the monoclonal antibody AUA1. Int J Cancer 1986;38:631-6.

2 Strnad J, Hamilton AE, Beavers LS, et al. Molecular cloning and characterization of a human adenocarcinoma/ epithelial surface antigen complementary DNA. Cancer Res 1989;49:314-17.

3 To A, Coleman DV, Dearnaley DP. Use of antisera to epithelial membrane antigen in serous effusions. J Clin Pathol 1981;34:1326-32.

4 Guzman J, Costabel U, Bross KJ, Wiehle U, Grunert F, Schaefer $\mathrm{HE}$. The value of the immunoperoxidase slide assay in the diagnosis of malignant pleural effusions in breast cancer. Acta Cytol 1988;32:1880-92.

5 Wick MR, Loy T, Mills SE, Legier JF, Manivel JC. Malignant epitheliod pleural mesothelioma versus peripheral pulmonary adenocarcinoma: A histochemical, ultrastructural and immunohistologic study of 103 cases. Hum Pathol 1990;21:759-66.

6 Pinto MM, Bernstein LH, Brogan DA, Criscuolo EM. Carcinoembrionic antigen in effusions: a diagnostic adjunct to cytology. Acta Cytol 1987;31:113-18.

7 Szpak CA, Soper JT, Thor A, Schlom J, Johnston WW. Detection of adenocarcinoma in peritoneal washings by Detection of adenocarcinoma in peritoneal washings by
staining with monoclonal antibody $\mathrm{B} 72 \cdot 3$. Acta Cytol 1989;33:205-14.

8 Daste G, Serre G, Mauduyt MA, Vincent C, Caveriviere $P$, Soleilhavoup JP. Immunophenotyping of mesothelial cells and carcinoma cells with monoclonal antibodies to cytokeratins, vimentin, CEA, EMA improves the cytodiagnosis of serous effusions. Cytopathology 1991;2:19-28.

9 Zuna RE, Mitchell ML. Cytologic findings in peritoneal washings associated with benign gynaecologic disease. Acta Cytol 1988;32:139-47.

10 Soosay GN, Griffiths M, Papadaki L, Happerfield L, Bobrow L. The differential diagnosis of epithelial type elial proliferation. J Pathol 1991;163:299-305. 